\title{
PROJECTIVE REPRESENTATIONS OF ABELIAN GROUPS
}

\author{
N. B. BACKHOUSE AND C. J. BRADLEY
}

\begin{abstract}
Let $\omega$ be a factor system for the locally compact abelian group $G$. Then we show that the finite-dimensional unitary irreducible projective representations of $G$, having factor system $\omega$, possess a common dimension $d(\omega)$. Using a characterisation of $d(\omega)$ as the index in $G$ of a maximal subgroup on which $\omega$ is symmetric we derive a formula for $d(\omega)$ in the case that $G$ is discrete and finitely generated.
\end{abstract}

1. Introduction. This paper developed out of a successful attempt to prove the following: if $D_{1}$ and $D_{2}$ are finite-dimensional irreducible unitary projective representations of a locally compact abelian group $G$, possessing the same factor system $\omega$, then $D_{1}$ and $D_{2}$ have the same dimension $d(\omega)$. Theorem 1 contains this result and states a fortiori that $D_{1}$ and $D_{2}$ can differ essentially only by a linear character of $G$.

Although in the general case it may not be possible to produce a formula for $d(\omega)$ in terms of $\omega$, some progress in this direction has been made in Theorem 3 with the identification of $d(\omega)$ as the index in $G$ of a maximal subgroup on which $\omega$ is symmetric. This is not just an incidental result for it plays a role in establishing a procedure for the evaluation of $d(\omega)$ in the special case that $G$ is discrete and finitely generated.

For the definitions and results appropriate to topological groups and their representations we refer the reader to Mackey [6] and [7], and in particular to Lemma 8.1 of [7] which is the key to the proof of Theorem 1. Although this lemma is only proved in the case of ordinary representations, to avoid duplication, we merely comment that most results about ordinary representations can be interpreted without essential alteration in terms of projective representations. We shall deal exclusively with finitedimensional unitary projective representations, and shall use the term $\omega$-representation to indicate the appropriate factor system and the term $\omega$-rep in irreducible cases. Also we assume that all groups are locally compact, though we do not restrict attention to abelian groups until Theorem 1.

Received by the editors May 24, 1971 and, in revised form, January 21, 1972. AMS 1970 subject classifications. Primary 22D12, 22B99, $20 \mathrm{C} 25$.

Key words and phrases. Locally compact abelian group, projective representation, factor system, Kronecker product, abelian group, finitely generated abelian group. 
2. The main theorem. Let $D$ be an $(\omega$-representation of $G$, then according to Mackey [6], $\bar{D}$, the adjoint of $D$, is an (i)-representation.

Concretely, if we consider $D$ as a matrix representation, then the matrices which correspond to $\bar{D}$ are the complex conjugates of those which correspond to $D$. Then we have the following result.

Lemma 1. Let $D_{1}$ and $D_{2}$ be $\omega$-representations of the group $G$. If $D_{2}$ is irreducible then $D_{1}$ contains $D_{2}$ as a direct summand if and only if the Kronecker product $D_{1} \otimes \bar{D}_{2}$ contains the trivial representation of $G$.

Proof. To establish this result we form the lifted representations $D_{1} \nearrow G^{\omega}$ and $D_{2}, G^{\omega}$, see Backhouse and Bradley [2], and apply directly Lemma 8.1 of Mackey [6]. Alternatively we note that the proof of Lemma 8.1 of [6] is valid word for word in the case of projective representations.

As an immediate application we have the following result, being a generalisation of a well-known property of ordinary representations.

Lemma 2. Let $D_{i}$ be an $\omega_{i}$-rep, having dimension $d_{i}$, of the group $G$, for $i=1,2,3$, where $\omega_{3}=\omega_{1} \omega_{2}$.

If $D_{3}$ is contained in $D_{1} \otimes D_{2}$ as a direct summand, then $\bar{D}_{2}$ is contained in $D_{1} \otimes \bar{D}_{3}$ and $\bar{D}_{1}$ is contained in $D_{2} \otimes \bar{D}_{3}$. Furthermore, $d_{3} \leqq d_{1} d_{2}, d_{2} \leqq d_{1} d_{3}$ and $d_{1} \leqq d_{2} d_{3}$.

Proof. The hypothesis, together with Lemma 1 , implies that $D_{1}(x)$ $D_{2} \otimes \bar{D}_{3}$ contains the trivial representation of $G$. Then noting that $D_{2}=\overline{\bar{D}}_{2}$ and with the commutativity and associativity of Kronecker products, the converse of Lemma 1 implies that $D_{1} \otimes \bar{D}_{3}, D_{2} \otimes \bar{D}_{3}$, contain $\bar{D}_{2}$ and $\bar{D}_{1}$, respectively, as required. Finally, the dimensionality constraints follow because the dimension of a subrepresentation cannot exceed that of its containing representation.

We now prove the main theorem.

THEOREM 1. Let $D_{1}$ and $D_{2}$ be two $\omega$-reps of the abelian group $G$. Then there exist a unitary transformation $U$ and a linear character $\chi$ where

$$
U^{-1} D_{1}(g) U=\chi(g) D_{2}(g),
$$

for all $g \in G$.

Proof. Let $D_{i}$ be of dimension $d_{i}$ for $i=1,2$. Since $\bar{D}_{2}$ is an $\overline{(i)}$-rep of $G$ it follows that $D_{1} \otimes \bar{D}_{2}$ is an ordinary representation of $G$, and since $G$ is abelian, contains at least one linear character $\%$. Now Lemma 2 implies that $d_{1} \leqq d_{2}$ and $d_{2} \leqq d_{1}$, and hence that $d_{1}=d_{2}$.

Lemma 2 tells us further that $\bar{D}_{1}$ is contained in $\bar{D}_{2} \otimes \bar{\chi}$, which being of equal dimension must be unitarily equivalent, and hence (4.1) follows. 
3. Maximal symmetric subgroups. Since the problem of describing the factor systems of an arbitrary abelian group is as yet unsolved, see, however, the work of Kleppner [4], it is unreasonable to expect a complete analysis of the dimension function $d(\omega)$. However, the knowledge of the existence of an $\omega$-rep $D$ of dimension $d(\omega)$ does impose certain conditions on ( $)$. For by definition

$$
\begin{aligned}
D\left(g_{1}\right) D\left(g_{2}\right) & =\omega\left(g_{1}, g_{2}\right) D\left(g_{1} g_{2}\right), \\
& =\omega\left(g_{1}, g_{2}\right) D\left(g_{2} g_{1}\right), \\
& =c\left(g_{1}, g_{2}\right) D\left(g_{2}\right) D\left(g_{1}\right),
\end{aligned}
$$

where $c\left(g_{1}, g_{2}\right)=\omega\left(g_{1}, g_{2}\right) / \omega\left(g_{2}, g_{1}\right)$, for all $g_{1}, g_{2} \in G$. Taking the determinant of both sides of (3.3) it follows that $c\left(g_{1}, g_{2}\right)$ is a $d(\omega)$ th root of unity for all $g_{1}, g_{2} \in G$. Further it has been shown by Kleppner [4] that $c$ is a bicharacter of $G$, that is, it is linear in each variable separately. We can at once deduce the following.

THEOREM 2. If $G$ is a divisible abelian group then $d(1)$ ) (if it is finite) is unity for every factor system $\omega$.

Proof. Since $G$ is divisible the bicharacter takes the value one throughout $G$. But then $\omega$ is symmetric on $G$, and so it follows from Theorem 2.1 of [3] that $\omega$ is equivalent to the trivial factor system. Hence all $\omega$-reps of $G$ are projectively equivalent to linear characters, which are of dimension one.

For example Theorem 2 holds if $G$ is a vector group. Hence the only $\omega$-reps of $R^{n}$ are the linear characters.

In general $\omega$ is not symmetric throughout $G$. However, since $\omega$ is symmetric on the identity subgroup, it follows from Zorn's lemma that there exists at least one maximal subgroup of $G$ on which $\omega$ is symmetric. It turns out that such a subgroup is relatively large, and indeed closely linked to the number $d(\omega)$.

THEOREM 3. If $M$ is a maximal subgroup of the abelian group $G$ on which $\omega$ is symmetric, then $M$ has index $d(\omega)$ in $G$.

Proof. We first claim that if $a_{1} \notin M$ then $M$ is of index $r_{1}$, where $2 \leqq r_{1} \leqq d(\omega)$, in the group $M\left(a_{1}\right)$ obtained by adjoining $a_{1}$ to $M$. For, if $t=a_{1}^{d(\omega)}$ then $c(t, g)=c\left(a_{1}, g\right)^{d(\omega)}=1$ for all $g \in M$. The bilinearity of $c$ implies that $\omega$ is symmetric on the group obtained by adjoining $t$ to $M$. The maximality of $M$ then implies that $t \in M$, and hence that $M$ is of index $r_{1}$ in $M\left(a_{1}\right)$, where $r_{1}$ is a divisor of $d(\omega)$ larger than unity.

If there exists $a_{2} \notin M\left(a_{1}\right)$, then it is evident that $M$ is of index $r_{2}$, where $4 \leqq r_{2} \leqq d(\omega)^{2}$, in the group $M\left(a_{1}, a_{2}\right)$ obtained by adjoining $a_{2}$ to $M\left(a_{1}\right)$. 
Continuing in this manner we decide to stop either if we find $M$ to be of index $\leqq d(\omega)$ in $G$ or if we find a subgroup $H$ of $G$ in which $M$ has index $>d(\omega)$.

Since $\omega$ is symmetric on $M$ it is possible to write it in the form

$$
\omega\left(g, g^{\prime}\right)=a(g) a\left(g^{\prime}\right) / a\left(g, g^{\prime}\right),
$$

for all $g, g^{\prime} \in M$, for some $T$-valued function $a$. It follows that an $\omega$-rep of $M$ is of the form $g \rightarrow a(g) b(g)$, where $b$ is a linear character of $M$. Since $M$ is of finite index in $G$ or $H$ we can apply the little $\omega$-group method, see Backhouse and Bradley [2], to this $\omega$-rep of $M$ to yield an $\omega$-rep of $G$ or $H$. As our initial representation is 1-dimensional its little $\omega$-group consists of all those $h \in G$ or $H$ which satisfy

$$
\omega(g, h) \omega\left(h^{-1}, g h\right)=\omega\left(h^{-1}, h\right),
$$

for all $g \in M$. Rewriting (3.5) we obtain

$$
(\omega(g, h) / \omega(h, g)) \omega\left(h^{-1}, h g\right) \omega(h, g)=\omega\left(h^{-1}, h\right),
$$

for all $g \in M$, and so

$$
(\omega(g, h) / \omega(h, g)) \omega(e, g) \omega\left(h^{-1}, h\right)=\omega\left(h^{-1}, h\right),
$$

using (2.5) of [2], and then $\omega(g, h)=\omega(h, g)$, for all $g \in M$, using (2.3) of [2]. The maximality of $M$ now implies that $h \in M$. It follows that the induced $\omega$-representation $a b \uparrow G$ or $H$ is irreducible. In the latter case $a b \uparrow H$ is of dimension $>d(\omega)$, which is a contradiction, since by restriction $H$ has $\omega$-reps of dimension $\leqq d(\omega)$. Otherwise $a b \uparrow G$ gives an $\omega$-rep of $G$, and since this is of dimension $d(\omega)$ it shows that $M$ is of index $d(\omega)$ in $G$.

COROLlaRY. Every $\omega$-rep of the abelian group $G$ is projectively equivalent to an induced linear character of some subgroup.

4. Finitely generated abelian groups. Unfortunately the characterisation of $d(\omega)$ given in Theorem 3 does not lead directly to a formula for $d(\omega)$. Even in cases where $\omega$ is easily described, for example if $G$ is finitely generated, it can be very difficult to find any maximal subgroups on which $\omega$ is symmetric. Indeed the following analysis of $d(\omega)$ for a finitely generated group shows just how complicated and unwieldy a maximal symmetric subgroup can be.

We first note that a finitely generated abelian group $G$ is a factor group of an appropriate free abelian group $T_{n}$ on a finite number of generators $n$. Furthermore since a factor system $\omega$ and an associated $\omega$-rep of $G$ can be lifted to a factor system and $\omega$-rep of $T_{n}$ it is sufficient in evaluating $d(\omega)$ to concentrate on $T_{n}$. 
If $\omega$ is a factor system of $T_{n}$, then without loss of generality it can be taken in the bilinear form as given in Theorem 4.1 of [2]. Thus, using a vector notation for the elements of $T_{n}$,

$$
\omega\left(\boldsymbol{t}, \boldsymbol{t}^{\prime}\right)=\exp \left(-2 \pi i \boldsymbol{t}^{t} G \boldsymbol{t}^{\prime}\right)
$$

for all $t, t^{\prime} \in T_{n}$, where $G$ is an $n \times n$ lower triangular or skew matrix. It is easy to show that $d(\omega)$ exists only if $G$ has rational entries. Suppose we change the basis of $T_{n}$, then with respect to this new basis the matrix determining $\omega$ is $U^{t} G U$, where $U$, representing a basis transformation, is a unimodular integer matrix. The idea is to choose $U$ in such a manner that as many as possible of the entries of $U^{t} G U$ are zero. In this connection we shall presently see the application of the following result.

LeMma 3. Let $C$ be an integer skew-symmetric $n \times n$ matrix. Then there exists a unimodular integer matrix $U$ such that $U^{t} C U$ is zero except for a certain number $r(2 r \leqq N)$ of $2 \times 2$ integer skew-symmetric matrices $E_{i}$ $(i=1,2, \cdots, r)$ placed along the diagonal. Furthermore it is possible to choose the matrices $E_{i}$ in such a way that the nonzero entries of $E_{i}$ divide the nonzero entries of $E_{i+1}$ for $1 \leqq i \leqq r-1$.

An inductive proof may be based on the case $n=3$ for which it is possible to explicitly calculate $U$. This result is due to Frobenius, and arose in his work on theta functions (see also Lang [5, p. 380]). The chief virtue of Lemma 3 is that we can explicitly determine the nonzero entries in $U^{t} C U$ without having to work out $U$ itself. This statement is based on the following result of $\mathrm{H}$. J. S. Smith (see for example Aitken and Turnbull [1]).

Lemma 4. If $B=P A Q$, where $P, A$ and $Q$, are $n \times n$ integer matrices, then the highest common factor of the $r \times r$ minors of $A$ divides the highest common factor of the $r \times r$ minors of $B$, for every $r \leqq n$.

COROllary. If $S=U^{t} C U$, where $U, C$, are $n \times n$ integer matrices with $U$ unimodular, then the highest common factors of the $r \times r$ minors of $C$ and $S$ are equal, for every $r \leqq n$.

We can now write down a procedure for evaluating $d(\omega)$.

1. Let the matrix $G$ which represents $\omega$ be in lower triangular form with its entries $G_{i j}=t_{i j / N i j}$, for $i>j$, as reduced fractions. Now add to $G$ the symmetric matrix whose $(i, j)$ entry is $-\frac{1}{2} G_{i j}$, for $1 \leqq i, j \leqq n$, to form the skew-symmetric matrix $A$.

2. If $N$ is the least common multiple of the $N_{i j}$ 's, then $A=(1 / 2 N) C$, where $C$ is a skew-symmetric integer matrix. 
3. According to Lemma 3 there exists a unimodular integer matrix $U$ such that

$$
U^{t} C U=E_{1} \oplus E_{2} \oplus \cdots \oplus E r \oplus O_{n-2 r},
$$

where

$$
E_{i}=\left(\begin{array}{cc}
0 & -a_{i} \\
a_{i} & 0
\end{array}\right)
$$

is a nonzero $2 \times 2$ integer matrix for $i=1,2, \cdots, r, r$ is the rank of $A$, and $a_{i} \mid a_{i+1}$ for $i=1,2, \cdots, r-1$.

4. Since a change of basis can have no effect on $d(\omega)$ we deduce from Theorem 3 that

$$
d(\omega)=\prod_{i=1}^{r} d_{i},
$$

where $d_{i}$ is the dimension corresponding to the factor system of $T_{2}$ obtained from the matrix $(1 / 2 N) E_{i}$. It follows from $\S 5$ of [2] that $d_{i}$ is $N /\left(N, a_{i}\right)$.

5. If $h_{i}$ is the highest common factor of the $(2 i-1) \times(2 i-1)$ minors of $C$, then for $i=1,2, \cdots, r$ we have

$$
h_{i}=a_{1}^{2} a_{2}^{2} \cdots a_{i-1}^{2} a_{i},
$$

and if $k_{i}$ is the highest common factor of the $2 i \times 2 i$ minors of $C$, then for $i=1,2, \cdots, r$ we have

$$
k_{i}=a_{1}^{2} a_{2}^{2} \cdots a_{i}^{2}
$$

If we put $k_{0}=1$, then

$$
\begin{aligned}
a_{i} & =h_{i} / k_{i-1}, \\
& =\left(k_{i} / k_{i-1}\right)^{1 / 2},
\end{aligned}
$$

for $i=1,2, \cdots, r$. Now we can write

$$
d(\omega)=N^{r} / \prod_{i=1}^{r}\left(N, a_{i}\right),
$$

where $a_{i}$ is given through (4.6) or (4.7) in terms of the integers $N G_{i j}$, $1 \leqq i, j \leqq n$.

ACKNOWLedgements. One of us (N. B. B.) would like to acknowledge several illuminating conversations with Miss Patricia Gard on the contents of this paper. He much appreciated the receipt of a maintenance grant for 1967-70 and a scholarship for 1967-70 from the British Science Research Council and Wolfson College, Oxford, respectively. 


\section{BIBLIOGRAPHY}

1. A. C. Aitken and H. W. Turnbull, An introduction to the theory of canonical matrices, Blackie, London, 1932.

2. N. B. Backhouse and C. J. Bradley, Projective representations of space groups. I. Translation groups, Quart. J. Math. Oxford Ser. (2) 21 (1970), 203-222. MR 41 \#5510.

3. N. B. Backhouse, Projective representations of space groups. II, Quart. J. Math. Oxford Ser. (2) 21 (1970), 277-295.

4. A. Kleppner, Multipliers on abelian groups, Math. Ann 158 (1965), 11-34. MR 30 \#4856.

5. S. Lang, Algebra, Addison-Wesley, Reading, Mass., 1965. MR 33 \#5416.

6. G. W. Mackey, Unitary representations of group extensions. I, Acta Math. 99 (1958), 265-311. MR 20 \#4789.

7. - Induced representations of locally compact groups. I, Ann. of Math (2) 55 (1952), 101-139. MR 13, 434.

Department of Applied Mathematics, University of Liverpool, Liverpool, ENGLAND

Department of Mathematics, Jesus College, Oxford, England 